\title{
The Development of the Extravascular Defibrillator with Substernal Lead Placement: A New Frontier for Device-Based Treatment of Sudden Cardiac Arrest
}

Amy Thompson ${ }^{1}$, Brett Atwater ${ }^{2}$, Lucas V.A. Boersma ${ }^{3}$, Ian Crozier $^{4}$, Gregory Engel ${ }^{5}$, J Rod Gimbel ${ }^{6}$, Bradley Knight ${ }^{7}$, Jaimie Manlucu ${ }^{8}$, Francis Murgatroyd ${ }^{9}$, David $\mathrm{O}^{\prime}$ Donnell ${ }^{10}$, Juergen Kuschyk ${ }^{11}$, and Paul DeGroot ${ }^{12}$

${ }^{1}$ Medtronic plc

${ }^{2}$ Inova Medical Group

${ }^{3}$ St. Antonius Hospital

${ }^{4}$ Christrchurch Hospital

${ }^{5}$ Palo Alto Medical Foundation

${ }^{6}$ Columbia Saint Mary's Hospital

${ }^{7}$ Northwestern University

${ }^{8}$ London Health Sciences Centre

${ }^{9}$ Kings College Hospital

${ }^{10}$ Austin and Repatriation Medical Center

${ }^{11}$ University Medical Center Mannheim

${ }^{12}$ Medtronic

December 6, 2021

\begin{abstract}
Introduction: The extravascular ICD (EV ICD) system with substernal lead placement is a novel non-transvenous alternative to current commercially available ICD systems. The EV ICD provides defibrillation and pacing therapies without the potential long-term complications of endovascular lead placement. Methods: This paper summarizes the development of the EV ICD, including the pre-clinical and clinical evaluations that have contributed to system and procedural refinements to date. Results: Extensive pre-clinical research evaluations and 4 human clinical studies with $>140$ combined acute and chronic implants have enabled the development and refinement of the EV ICD system, currently in worldwide pivotal study. Conclusion: The EV ICD may represent a clinically valuable solution in protecting patients from sudden cardiac death while avoiding the long-term consequences of transvenous hardware. The EV ICD offers advantages over transvenous and subcutaneous systems by avoiding placement in the heart and vasculature; relative to subcutaneous systems, EV ICD requires less energy for defibrillation, enabling a smaller device, and provides pacing features such as anti-tachycardia and asystole pacing in a single system.
\end{abstract}

\section{Hosted file}

EV ICD History Manuscript JCE.docx available at https://authorea.com/users/403690/ articles/548257-the-development-of-the-extravascular-defibrillator-with-substernal-leadplacement-a-new-frontier-for-device-based-treatment-of-sudden-cardiac-arrest 